\title{
Correlation Between Glucose Metabolism Parameters Derived From FDG and Tumor TNM Stages and Metastasis-Associated Proteins in Colorectal Carcinoma Patients
}

\section{Mingyu Zhang}

Capital Medical University Affiliated Beijing Friendship Hospital

\section{Jigang Yang}

Capital Medical University Affiliated Beijing Friendship Hospital Department of nuclear medicine

\section{Hao Jiang}

Second Affiliated Hospital of Harbin Medical University Department of Radiology

\section{Huijie Jiang ( $\sim$ jhjemail@163.com )}

Second Affiliated Hospital of Harbin Medical University Department of Radiology

\section{Zhenchang Wang}

Capital Medical University Affiliated Beijing Friendship Hospital Department of Radiology

\section{Original research}

Keywords: Positron emission tomography, Colorectal carcinoma, Fluorodeoxyglucose, Glucose transporter 1, Metastasis-associated in colon cancer 1

Posted Date: August 24th, 2020

DOl: https://doi.org/10.21203/rs.3.rs-60143/v1

License: (c) (i) This work is licensed under a Creative Commons Attribution 4.0 International License. Read Full License

Version of Record: A version of this preprint was published at BMC Cancer on March 9th, 2021. See the published version at https://doi.org/10.1186/s12885-021-07944-z. 


\section{Abstract}

Background The aim of this study was to investigate the relationship between multiple metabolism parameters derived from FDG and tumor TNM stages as well as tumor metastasis-associated protein of GLUT-1 and MACC1 in colorectal carcinoma (CRC).

Methods 38 patients (24 male and 14 female) with primary CRC confirmed by elective surgery pathological, who also underwent ${ }^{18}$ F-FDG PET/CT scans between 2017 and 2019 were included in this study. Tumor T, N and M classification according to the 7th American Joint Committee on Cancer (AJCC). ${ }^{18} \mathrm{~F}$-FDG parameters of SUVmax》 SUVmean, TLG and MTV were measured by drawing a region of interest on the primary lesions. The expression of GLUT-1 and MACC1 was quantified by immunohistochemical and the correlation between metabolism parameters and tumor biomarkers were analyzed.

Results According to our analysis, the ${ }^{18} \mathrm{~F}-\mathrm{FDG}$ parameters of SUVmean was significantly correlated with tumor M status $(\mathrm{P}=0.000)$ of primary CRC. The primary tumor lesion with higher SUVmax, TLG and MTV values prone to a highT status ( $P=0.002,0.002$ and 0.001 , respectively). The high expression of GLUT-1/MACC1 were more frequently involved with T3-4 stage and poorly differentiated in CRC patients. Multivariate analysis found that the expression of GLUT-1 protein was correlated with SUVmax and MTV $\left(R^{2}=0.42, P=0.013\right.$ and 0.004 , respectively), moreover, the expression of MACC1 protein was correlated with $T L G\left(R^{2}=0.372, P=0.000\right)$.

Conclusion Glucose metabolism parameters derived from FDG provides a noninvasive assessment of $\mathrm{M}$ status and T status in CRC patients. The expression of GLUT-1 and MACC1 was associated with ${ }^{18} \mathrm{~F}$-FDG uptake in CRC patients.

\section{Background}

Colorectal carcinoma (CRC) is the third most commonly diagnosed malignancy and the fourth cause of cancer-related death worldwide [1, 2]. Recently, cancer registry data show that in some high-income countries an increasing incidence of $\mathrm{CRC}$ in adults younger than 50 years[3]. Changes in dietary patterns which is called as western lifestyle are considered to be high risk factors for CRC. Early evaluation of tumor status contributes to the development of therapeutic management and prognostic prediction.

Glucose transporter-1 (GLUT-1) is one of a membrane transport proteins among glucose transporter family. It has been found to be overexpressed in variety of malignancies including CRC, and expression level of GLUT-1 was correlated with tumor metastatic potential and poor prognosis [4, 5]. In previous studies, the expression of GLUT-1 was considered as an independent prognostic indicators of worse outcome in T1-2 stage CRC[6]. MACC1 is another promising biomarker in early diagnosis and predict metastasis potential of CRC [7]. Accumulating evidence of in vivo and in vitro studies indicated that high expression of MACC1 was strongly associated with tumor formation, metastases development and poor prognosis [8, 9]. Therefore, GLUT-1 and MACC1 may established as a suitable biomarkers for predicting the tumor TNM stage of CRC patients, in order to improve individualized therapy regimen in CRC.

Conventional evaluation of tumor staging relies on electronic colonoscopy, multiple sites CT and MRI scanning and immunohistochemistry evaluation under biopsy. It is difficult to reflect the overall appearance of the tumor and increase the complexity of the examination. To integration of various technologies to obtain optimal diagnostic approach is necessary for clinical treatment. Clinical application of ${ }^{18} \mathrm{~F}-\mathrm{FDG}$ PET/CT was considered to be an important method to diagnosis, staging and monitor the therapeutic response of CRC $[10,11]$. The parameters of ${ }^{18} \mathrm{~F}$ FDG reflect the tracer uptake by tumor cells and provides objective data on tumor glucose metabolism. Recently, the correlation between radio-parameters derived from FDG-PET and tumor biomarkers has been extensively investigated, 
especially GLUT-1, high expression of GLUT-1 is identified as promote the FDG uptake in wide variety of solid tumors [12]. However, the role of MACC1 in contribute to FDG uptake needs further exploration.

In this study, we retrospectively assessed the feasibility of multiple radio-parameters derived from FDG in predicting tumor stages as well as determine the correlation between GLUT-1/MACC1 and TNM stage and tumor glucose metabolism of CRC. Our findings may provide a useful image data in preoperative evaluation of CRC status.

\section{Patients And Methods}

\section{Patient selection}

We retrospectively analyzed 38 patients (male 24 and female 14) histologically diagnosed with CRC, who underwent surgery excision between January 2017 and July 2019, who also underwent ${ }^{18}$ F-FDG PET/CT scan prior to surgery. Inclusion criteria: 1) All the patients were diagnosed primary CRC for the first time without any treatment; 2) Patient has complete clinical, pathological and imaging data; 3) Patient did not merge with other parts of the primary tumor; 4) Patient performs PET / CT scan for the first time. Clinicopathological data including gender, age, T status, N status, M status, Differentiation status were obtained. Primary lesion specimens were obtained for immunohistochemical analysis.

\section{${ }^{18}$ F-FDG PET/CT protocol and imaging analysis}

All patients required fasting for more than 6 hours before PET/CT examination and blood glucose levels were measured by a dedicated electronic glucometer to controlled levels of $<10.0 \mathrm{mmol} / \mathrm{L}$. The whole-body PET/CT was conducted with a three-dimensional mode using a PET-CT scanner (Biograph $64 \mathrm{HR}$, Siemens Medical Solutions). ${ }^{18} \mathrm{~F}-$ FDG (approximately 259-370 MBq) was injected intravenously $1 \mathrm{~h}$-post the scanning. Patients in supine position with

the arms up were scanned. Firstly, CT scan were performed to (120kV, 200mA and slice thickness of $3 \mathrm{~mm})$ attenuation correction and anatomical localization, and the PET scan was performed in a three-dimensional mode with a 2.5 minutes per bed position and generally scans 6-8beds. PET attenuation correction was based on CT data and PET images were reconstructed using an iterative algorithm.

For FDG semi-quantitative analysis, a three-dimensional region of interest (ROI) was manually drawn on the primary lesion and analyzed on Advantage Workstation. The maximum/mean standard uptake value (SUVmax/SUVmean), metabolic tumor volume (MTV), and total lesion glycolysis (TLG) of the primary CRC lesions were then obtained and recorded. A maximal pixel value of $40 \%$ was used for ROI measurements.

\section{Immunohistochemical analysis}

Paraffin-embedded CRC specimens were cut into 4mm-thick sections and immunohistochemical staining was performed as previously described [13]. Briefly, the slides were incubated with anti-GLUT-1 (1:100, Abcam) or antiMACC1 $(1: 80, \mathrm{Abcam})$ at $4^{\circ} \mathrm{C}$ overnight. Next, the slides were incubated with horseradish peroxidase-labeled secondary antibody (Boster, Wuhan, China) at room temperature followed by counterstaining with hematoxylin. The staining was observed under a BX53 Olympus microscope (Olympus, Japan) at magnification 200x. A brown-yellow staining was defined as positive expression. GLUT-1 or MACC1 protein were quantitated by Image-J software (NIH, Bethesda, MD, USA).

\section{Statistical analysis}


All data were expressed as mean \pm standard deviation. Statistical analyses were performed with SPSS version 19.0 (IBM, Armonk, NY, USA). Multiple stepwise regression test was used to evaluate the relationship between tumor markers or radioparameters and clinicopathologic parameters, as well as the relationship between radioparameters and tumor markers. The receiver operating characteristic (ROC) curve was performed to evaluate the diagnostic accuracy of SUVmax, SUVmean, TLG or MTV cut-off values that best predicted tumor T status or M status. A P value $<0.05$ was considered statistically significant.

\section{Results}

\section{Patient population}

Totally, thirty eight patients underwent whole-body ${ }^{18}$ F-FDG PET/CT before surgery between 2017 and 2019 were included in this study. The diagnosis of primary CRC lesions were confirmed by pathology. The majority were male (24, $63.2 \%)$ and the average age was 63.79. Twenty eight (73.7\%) patients with T3 stage. Four patients with T4 stage (10.5\%). Only six patients with T1 and T2 stage (15.8\%). Fifteen (39.5\%) patients were existed synchronous distant metastasis at time of diagnosis. The majority of pathologic subtype was moderately differentiated (76.3\%), followed by poorly differentiated (18.4\%) and well differentiated (5.3\%). Thirteen (34.2\%) patients had on lymph metastasis, moreover twenty-five (65.8\%) patients had lymph metastasis (AJCC 7th). Details of patients' characteristics are summarized in Table 1.

\section{Relationship between TNM stages and the expression of GLUT-1 and MACC1 in CRC}

GLUT-1 and MACC1 play an important role in tumor progression, we next to investigated the relationship between GLUT-1/MACC1 and tumor T $\triangle N$ and $M$ status in primary CRC lesions. For immunohistochemical staining, the positive expression of GLUT-1 was localized to the membranous of CRC cells, while MACC1 protein was localized to the cytoplasm. The expression of MACC1 protein was higher than GLUT-1 in the same patient. Multivariate analysis shown a significant relationship between GLUT-1/MACC1 expression and T status $\triangle \mathrm{P}=0.006$ and 0.002 Q $\mathrm{B}$ also between GLUT1/MACC1 expression and differentiated degree ( $P=0.009$ and 0.014$)$. CRC patients with high T-status and poorly differentiated tended to have higher GLUT-1 and MACC1 expression levels.

\section{Relationship between TNM stages and FDG parameters in CRC}

All the primary lesions of CRC which quantitative by ROI measurements with a SUVmax greater than 2.5 was identified as positive. The SUVmax, SUVmean, TLG and MTV values in patients with different T, N, M or D (differentiation) status were calculated. According to our multivariate analysis, both SUVmax, TLG and MTV values had a statistical correlated with $T$ status ( $P=0.002,0.002$ and 0.001 , respectively), meanwhile SUVmean values had a statistical correlated with $M$ status $(P=0.000)$ of primary CRC. ROC analysis showed that the area under the curve (AUC) was 0.838 $(P=0.029)$, $0.819(P=0.01), 0.941(P=0.04)$ and $0.96(P=0.003)$ for SUVmax, SUVmean, TLG and MTV, respectively. SUVmax cut-off value of 18.75 showed sensitivity of $100 \%$, specificity of $70.6 \%$, MTV cut-off value of 30.5 showed sensitivity of $100 \%$, specificity of $82.4 \%$ and TLG cut-off value of 275.85 showed sensitivity of $100 \%$, specificity of $88.2 \%$ in distinguish CRC T3 from T4 status. A SUVmean cut-off value of 11.7 showed sensitivity of $66.7 \%$, specificity of $87 \%$ in distinguish CRC M status.

\section{Relationship between GLUT-1/MACC1 expression and ${ }^{18} \mathrm{~F}-\mathrm{FDG}$ uptake in primary CRC lesions}

As GLUT-1 and MACC1 were a promising biomarkers to distinguish tumor progression and malignant degree. We next to investigate whether GLUT-1/MACC1 expression can reflect tumor glycometabolism. Through multivariate analysis 
the expression of GLUT-1 protein was correlated with SUVmax and MTV $\left(R^{2}=0.42, P=0.013\right.$ and 0.004 , respectively), moreover, the expression of MACC1 protein was correlated with TLG $\left(R^{2}=0.372, P=0.000\right)$. The expression levels of GLUT-1 in CRC primary lesion may better reflect the character tumor glucose metabolism volume and MACC1 may reflect total lesion glycolysis (Fig 1 and 2).

\section{Discussion}

Imaging technology is the most commonly used tool in the evaluation for the initial diagnosis, staging, restaging and monitoring treatment of CRC, especially those cannot traversed colonoscopically. MRI has a significant advantage in assessing $T$ staging of primary rectal cancer because of the capacity to differentiate the intestinal wall laminar structure [14]. However, evaluation of $\mathrm{N}$ and $\mathrm{M}$ stage in patients with $\mathrm{CRC}$ using conventional procedures was still present challenges until the emergence of whole-body ${ }^{18} \mathrm{~F}-\mathrm{FDG}$ PET/CT. PET/CT integrate morphologic and molecular data to detecting tumor biological behavior, furthermore provide lymph nodes and distant metastases information.

In this study, we detected the semi-quantitative (SUVmax囚SUVmean, TLG and MTV) derived from FDG and the expression of tumor markers (GLUT-1 and MACC1) in patients with different stages of CRC which consistent with pathological results. Our finding demonstrated that a significant correlation was found between SUVmax, TLG and MTV values and T status ( $P=0.002,0.002$ and 0.001 , respectively), meanwhile another correlation was found between SUVmean values and $M$ status $(P=0.000)$ of primary CRC. The area under the curve (AUC) of SUVmax, SUVmean, TLG and MTV was $0.838,0.819,0.941$ and 0.96 , respectively. For distinguish T3 status from T4 of CRC, the SUVmax cut-off value of 18.75 showed sensitivity of $100 \%$, specificity of $70.6 \%$, SUVmean cut-off value of 11.7 showed sensitivity of $66.7 \%$, specificity of $87 \%$ and TLG cut-off value of 275.85 showed sensitivity of $100 \%$, specificity of $88.2 \%$. For distinguish M status of CRC, MTV cut-off value of 30.5 showed sensitivity of $100 \%$, specificity of $82.4 \%$. Those results suggested that enhanced glucose uptake is associated with malignant tumor progression, similarly FDG parameters data provide metabolic characteristic that have the capacity to distinguish tumor T stage and M stage of CRC. The same results consistent with previous study, it is considered that SUV value of FDG PET/CT is more suitable for assessment tumor stage compared with CT and MRI, and the combination of colonoscopy will be more beneficial for CRC staging $[15,16]$. Clinically, PET/CT scanning is an essential project to evaluate $\mathrm{T}, \mathrm{N}$ and $\mathrm{M}$ stage preoperative in patients with CRC.

In previous meta-analysis, overexpressed GLUT-1 was considered to be associated with clinical features including lymph node metastasis, T stage, higher Dukes stage and disease-free survival of CRC [4]. Overexpressed MACC1 was contribute to the transformation of malignant degree and metastatic potential of CRC, primarily for adenoma transform into Tis further transform into invasive $\mathrm{CRC}[7,17]$. Our results demonstrated a significant relationship between GLUT-1/MACC1 expression and T status $\otimes P=0.006$ and 0.002 खdifferentiated degree $(P=0.009$ and 0.014$)$, CRC patients with high T-status and poorly differentiated tended to have higher GLUT-1/MACC1 expression levels. Our data reveal that GLUT-1 and MACC1 has a prognostic capacity for stratified tumor pathological factors ( $T$ status and differentiated degree). Furudoi, A. et al. study suggest that the GLUT-1 expression at the deepest invasive site was significantly with clinicopathologic features including lymph node and Duke's stage [18]. Our finding also consistent with Aifen Lin's study, shown that high expression of MACC1 was correlation with T stage of CRC and was a valuable prognostic and risk stratification biomarker of CRC[17]. Shirahata A. et al. found that MACC1 expression was significantly correlated with peritoneal dissemination and higher TNM stage of CRC [19]. Inconsistent with previous study, the average expression of GLUT-1 and MACC1 were higher in M1 and N2 specimen but did not reach the statistical difference which may due to the limitation of small sample volume in our experiment. Moreover our study found that the expression of MACC1 was higher than GLUT-1 in the same patient, MACC1 protein may be superior to GLUT-1 in predicting the biological characteristics of CRC.

Page 5/14 
Subsequently, our finding revealed the relationship between biomarker and radio-parameters derived from FDG PET. Our multivariate analysis shown a significantly correlation between GLUT- 1 and SUVmax, MTV $\left(R^{2}=0.42, P=0.013\right.$ and 0.004 , respectively), another correlation was found between MACC1 and TLG $\left(R^{2}=0.372, P=0.000\right)$. In most studies, SUVmax was the most commonly used parameter to evaluate FDG uptake, however, tumor volumetric parameters of FDG such as MTV and TLG provides more comprehensive informations on tumor metabolism and reflect tumor biological characteristics more accurately [20]. In our study, the volumetric parameters of MTV and TLG show a power in evaluating the expression of GLUT-1 and MACC1 in CRC specimen. The mechanism of FDG uptake was acknowledged to be mainly mediated by GLUT family, and GLUT-1 was one of the most important contributor. Enhanced FDG uptake reflect the heterogeneity of glucose metabolism. The correlation between GLUT-1 and FDG uptake was found in multiple malignancies such as breast cancer[21], lung cancer[22], cervical cancer[23]『pancreatic cancer[24] and colorectal cancer[12] et al. However, Ran Hong's study show that the GLUT-1 expression was not associated with FDG uptake, the discrepancy between two studies may be caused by differences in FDG parameters, only one radio-parameter was included in Ran Hong's research [25]. There was few study regarded to the relationship between MACC1 and FDG uptake, the present study demonstrated a statistical correlation between MACC1 expression and TLG of CRC patients for the first time. The expression levels of MACC1 in primary CRC lesion may better reflect the character of tumor total lesion glycolysis. This correlation may explained as high MACC1 levels is related to tumor progression, overexpressed MACC1 enhanced the tumor Warburg effect which increasing the glucose metabolism activity of CRC $[17,26]$. Consistent with Jing Liu study, demonstrates that MACC1 overexpression promotes ${ }^{18}$ F-FDG uptake in mice bearing NCl-N87 xenograft [27]. To investigating the relationship between biomarkers and FDG uptake will deeply understanding tumor biological behavior and lead to a clearer interpretation of PET imaging.

\section{Conclusion}

In summary, the present study demonstrated the feasibility of glucose metabolism parameters derived from FDG to predicting $T$ status and M status of CRC patients noninvasively. A high levels of GLUT-1 and MACC1 expression show a predict capacity for stratified tumor pathological factors (T status and differentiated degree). Moreover, overexpression of GLUT-1 and MACC1 may promote the glucose metabolism activity of CRC.

\section{Abbreviations}

CRC: Colorectal carcinoma

CLM: colorectal liver metastases

PET囚Positron emission tomography

MACC1囚Metastasis-associated in colon cancer 1

GLUT-1هGlucose transporter 1

MTV: Metabolic tumor volume

TLG: Total lesion glycolysis

\section{Declarations}

\section{Ethics approval and consent to participate}


This study was approved by the Ethics Committee of the Second Affiliated Hospital of Harbin Medical University (KY2018-215). All the procedures were conducted according to protocol approved by the ethics committee of the second affiliated hospital of Harbin medical university.

\section{Consent for publication}

Not applicable

\section{Availability of Data and Materials}

The datasets used and/or analysed during the current study are available from the corresponding author on reasonable request

\section{Competing interests}

All the authors declare that they have no conflict of interest.

\section{Funding}

This study was supported by grants from the National Key Research and Development Program of China (2019YFC0118100) ; National Natural Science Foundation of China (81671760, 81873910 and 61931013), Scientific Research Transformation Special Fund of Heilongjiang Academy of Medical Sciences $₫ 2018415 \otimes \bigotimes$ Scientific Research Project of Health and Family Planning Commission of Heilong jiang Province(201812 and 201622)区No. [2015] 160 from Beijing Scholars Program; No. YYZZ201919 from Beijing Friendship Hospital Seed Project, Capital Medical University; No. 2020-Z2-022 from Beijing Postdoctoral Research Foundation.

\section{Authors' contributions}

ZMY, JHJ and WZC contributed to conception and design; ZMY, JHJ, YJG, JH and WZC contributed to acquisition of data, or analysis and interpretation of data; ZMY, JHJ, JH, YJG and WZC have been involved in drafting the manuscript or revising it critically for important intellectual content; all authors have given final approval of the version to be published.

\section{Acknowledgments}

None

\section{References}

1. Brenner H, Kloor M, Pox CP: Colorectal cancer. The Lancet. 2014 383(9927):1490-1502.

2. Center MM, Jemal A, Ward E: International trends in colorectal cancer incidence rates. Cancer Epidemiol Biomarkers Prev. 2009, 18(6):1688-1694.

3. Siegel RL, Torre LA, Soerjomataram I, Hayes RB, Bray F, Weber TK, Jemal A: Global patterns and trends in colorectal cancer incidence in young adults. Gut. 2019, 68(12):2179-2185.

4. Yang J, Wen J, Tian T, Lu Z, Wang Y, Wang Z, Wang X, Yang Y: GLUT-1 overexpression as an unfavorable prognostic biomarker in patients with colorectal cancer. Oncotarget. 2017, 8(7):11788-11796.

5. Graziano F, Ruzzo A, Giacomini E, Ricciardi T, Aprile G, Loupakis F, Lorenzini P, Ongaro E, Zoratto F, Catalano V et al: Glycolysis gene expression analysis and selective metabolic advantage in the clinical progression of colorectal cancer. Pharmacogenomics J. 2017, 17(3):258-264. 
6. Lastraioli E, Bencini L, Bianchini E, Romoli MR, Crociani O, Giommoni E, Messerini L, Gasperoni S, Moretti R, Di Costanzo F et al: hERG1 Channels and Glut-1 as Independent Prognostic Indicators of Worse Outcome in Stage I and II Colorectal Cancer: A Pilot Study. Transl Oncol. 2012, 5(2):105-112.

7. Ren B, Zakharov V, Yang Q, McMahon L, Yu J, Cao W: MACC1 is related to colorectal cancer initiation and earlystage invasive growth. Am J Clin Pathol. 2013, 140(5):701-707.

8. Stein U, Walther W, Arlt F, Schwabe H, Smith J, Fichtner I, Birchmeier W, Schlag PM: MACC1, a newly identified key regulator of HGF-MET signaling, predicts colon cancer metastasis. Nature medicine. 2009, 15(1):59-67.

9. Schmid F, Wang Q, Huska MR, Andrade-Navarro MA, Lemm M, Fichtner I, Dahlmann M, Kobelt D, Walther W, Smith $J$ et al: SPON2, a newly identified target gene of MACC1, drives colorectal cancer metastasis in mice and is prognostic for colorectal cancer patient survival. Oncogene. 2016, 35(46):5942-5952.

10. Gu J, Yamamoto H, Fukunaga H, Danno K, Takemasa I, Ikeda M, Tatsumi M, Sekimoto M, Hatazawa J, Nishimura T et al: Correlation of GLUT-1 overexpression, tumor size, and depth of invasion with 18F-2-fluoro-2-deoxy-Dglucose uptake by positron emission tomography in colorectal cancer. Dig Dis Sci. 2006, 51(12):2198-2205.

11. Park IJ, Kim HC, Yu CS, Ryu MH, Chang HM, Kim JH, Ryu JS, Yeo JS, Kim JC: Efficacy of PET/CT in the accurate evaluation of primary colorectal carcinoma. Eur J Surg Oncol. 2006, 32(9):941-947.

12. Meyer HJ, Wienke A, Surov A: Associations between GLUT expression and SUV values derived from FDG-PET in different tumors-A systematic review and meta analysis. PLoS One. 2019, 14(6):e0217781.

13. Ding Z, Yang L, Xie X, Xie F, Pan F, Li J, He J, Liang H: Expression and significance of hypoxia-inducible factor-1 alpha and MDR1/P-glycoprotein in human colon carcinoma tissue and cells. J Cancer Res Clin Oncol. 2010, 136(11):1697-1707.

14. Kijima S, Sasaki T, Nagata K, Utano K, Lefor AT, Sugimoto H: Preoperative evaluation of colorectal cancer using CT colonography, MRI, and PET/CT. World J Gastroenterol. 2014, 20(45):16964-16975.

15. Taylor SA, Bomanji JB, Manpanzure L, Robinson C, Groves AM, Dickson J, Papathanasiou ND, Greenhalgh R, Ell PJ, Halligan S: Nonlaxative PET/CT colonography: feasibility, acceptability, and pilot performance in patients at higher risk of colonic neoplasia. J Nucl Med. 2010, 51(6):854-861.

16. Veit P, Kuhle C, Beyer T, Kuehl H, Herborn CU, Borsch G, Stergar H, Barkhausen J, Bockisch A, Antoch G: Whole body positron emission tomography/computed tomography (PET/CT) tumour staging with integrated PET/CT colonography: technical feasibility and first experiences in patients with colorectal cancer. Gut. 2006, 55(1):68-73.

17. Lin A, Zhang X, Zhang RL, He XF, Zhang JG, Yan WH: Prognostic and Risk Stratification Value of Lesion MACC1 Expression in Colorectal Cancer Patients. Front Oncol. 2019, 9:28.

18. Furudoi A, Tanaka S, Haruma K, Yoshihara M, Sumii K, Kajiyama G, Shimamoto F: Clinical significance of human erythrocyte glucose transporter 1 expression at the deepest invasive site of advanced colorectal carcinoma. Oncology. 2001, 60(2):162-169.

19. Shirahata A, Shinmura K, Kitamura Y, Sakuraba K, Yokomizo K, Goto T, Mizukami H, Saito M, Ishibashi K, Kigawa G et al: MACC1 as a marker for advanced colorectal carcinoma. Anticancer Res. 2010, 30(7):2689-2692.

20. Bahri H, Laurence L, Edeline J, Leghzali H, Devillers A, Raoul JL, Cuggia M, Mesbah H, Clement B, Boucher E et al: High prognostic value of 18F-FDG PET for metastatic gastroenteropancreatic neuroendocrine tumors: a long-term evaluation. J Nucl Med. 2014, 55(11):1786-1790.

21. Wuest M, Hamann I, Bouvet V, Glubrecht D, Marshall A, Trayner B, Soueidan OM, Krys D, Wagner M, Cheeseman C et al: Molecular Imaging of GLUT1 and GLUT5 in Breast Cancer: A Multitracer Positron Emission Tomography Imaging Study in Mice. Molecular pharmacology. 2018, 93(2):79-89. 
22. El-Chemaly S, Malide D, Yao J, Nathan SD, Rosas IO, Gahl WA, Moss J, Gochuico BR: Glucose transporter-1 distribution in fibrotic lung disease: association with [(1)(8)F]-2-fluoro-2-deoxyglucose-PET scan uptake, inflammation, and neovascularization. Chest. 2013, 143(6):1685-1691.

23. Tong SY, Lee JM, Ki KD, Choi YJ, Seol HJ, Lee SK, Huh CY, Kim GY, Lim SJ: Correlation between FDG uptake by $\mathrm{PET} / \mathrm{CT}$ and the expressions of glucose transporter type 1 and hexokinase II in cervical cancer. International journal of gynecological cancer : official journal of the International Gynecological Cancer Society. 2012, 22(4):654-658.

24. Chikamoto A, Inoue R, Komohara Y, Sakamaki K, Hashimoto D, Shiraishi S, Takamori H, Yamashita YI, Yoshida N, Yamanaka T et al: Preoperative High Maximum Standardized Uptake Value in Association with Glucose Transporter 1 Predicts Poor Prognosis in Pancreatic Cancer. Ann Surg Oncol. 2017, 24(7):2040-2046.

25. Hong R, Lim SC: (1)(8)F-fluoro-2-deoxyglucose uptake on PET CT and glucose transporter 1 expression in colorectal adenocarcinoma. World J Gastroenterol. 2012, 18(2):168-174.

26. Wang L, Wu Y, Lin L, Liu P, Huang H, Liao W, Zheng D, Zuo Q, Sun L, Huang N et al: Metastasis-associated in colon cancer-1 upregulation predicts a poor prognosis of gastric cancer, and promotes tumor cell proliferation and invasion. Int J Cancer. 2013, 133(6):1419-1430.

27. Liu J, Pan C, Guo L, Wu M, Guo J, Peng S, Wu Q, Zuo Q: A new mechanism of trastuzumab resistance in gastric cancer: MACC1 promotes the Warburg effect via activation of the PI3K/AKT signaling pathway. J Hematol Oncol. $2016,9(1): 76$.

\section{Tables}

Table 1 Patient and tumor characteristics. 


\begin{tabular}{|c|c|c|}
\hline Characteristic & No. of patients & Value (\%] \\
\hline Gender, male/female & $38 \rrbracket 24 / 14 \rrbracket$ & $63.2 / 36.8$ \\
\hline \multicolumn{3}{|l|}{ Agelyears $\square$} \\
\hline Mean $\pm S D$ & & $63.79 \pm 13.94$ \\
\hline Range & & $32-89$ \\
\hline \multicolumn{3}{|l|}{ T stages } \\
\hline $\mathrm{T} 1 / \mathrm{T} 2$ & 6 & 15.8 \\
\hline T3 & 28 & 73.7 \\
\hline $\mathrm{T} 4$ & 4 & 10.5 \\
\hline \multicolumn{3}{|l|}{ N stages } \\
\hline NO & 13 & 34.2 \\
\hline N1 & 13 & 34.2 \\
\hline N2 & 12 & 31.6 \\
\hline \multicolumn{3}{|l|}{ M stage } \\
\hline MO & 23 & 60.5 \\
\hline M1 & 15 & 39.5 \\
\hline \multicolumn{3}{|l|}{ Differentiation } \\
\hline Well & 2 & 5.3 \\
\hline Moderate & 29 & 76.3 \\
\hline Poorly & 7 & 18.4 \\
\hline
\end{tabular}

The clinical stages shown in this table were categorized according to the AJCC (7th guideline)

Table 2 Multivariate regression analysis of the relationship between TNM status and the expression of GLUT-1 and MACC1 of 38 patients with CRC 


\begin{tabular}{|c|c|c|c|c|c|}
\hline Variable & No. of patients & GLUT-1 & $P$ value & MACC1 & $P$ value \\
\hline Gender & 38 & & 0.976 & & 0.217 \\
\hline Male & 24 & $37.87 \pm 12.39$ & & $58.91 \pm 17.03$ & \\
\hline Female & 14 & $35.71 \pm 9.17$ & & $51.22 \pm 11.70$ & \\
\hline T status & & & 0.006 & & 0.002 \\
\hline $1 / 2$ & 6 & $23.44 \pm 4.91$ & & $41.42 \pm 15.54$ & \\
\hline 3 & 28 & $37.85 \pm 9.50$ & & $55.98 \pm 12.10$ & \\
\hline 4 & 4 & $52.13 \pm 4.93$ & & $78.76 \pm 12.46$ & \\
\hline N status & & & 0.072 & & 0.711 \\
\hline NO & 13 & $30.19 \pm 6.40$ & & $50.82 \pm 15.21$ & \\
\hline N1 & 13 & $37.38 \pm 10.46$ & & $53.12 \pm 15.07$ & \\
\hline N2 & 12 & $44.20 \pm 12.22$ & & $64.98 \pm 13.63$ & \\
\hline M status & & & 0.682 & & 0.843 \\
\hline MO & 23 & $34.98 \pm 11.60$ & & $52.68 \pm 15.06$ & \\
\hline M1 & 15 & $40.29 \pm 10.20$ & & $61.29 \pm 15.36$ & \\
\hline Differentiation & & & 0.009 & & 0.014 \\
\hline Well & 2 & $22.15 \pm 7.64$ & & $29.17 \pm 8.73$ & \\
\hline Moderate & 29 & $35.18 \pm 9.27$ & & $54.39 \pm 11.62$ & \\
\hline Poorly & 7 & $49.19 \pm 10.27$ & & $70.77 \pm 18.59$ & \\
\hline
\end{tabular}

Table 3 Multivariate analysis of the relationship between T, N and $\mathrm{M}$ status and ${ }^{18} \mathrm{~F}$-FDG parameters of 38 patients with CRC 


\begin{tabular}{|c|c|c|c|c|c|c|c|c|}
\hline \multirow[t]{2}{*}{ Variable } & \multicolumn{2}{|l|}{ SUVmax } & \multicolumn{2}{|l|}{ SUVmean } & \multicolumn{2}{|l|}{ TLG } & \multicolumn{2}{|l|}{ MTV } \\
\hline & Mean $\pm S D$ & $\begin{array}{l}P \\
\text { value }\end{array}$ & Mean $\pm S D$ & $\begin{array}{l}\mathrm{P} \\
\text { value }\end{array}$ & Mean $\pm S D$ & $\begin{array}{l}\mathrm{P} \\
\text { value }\end{array}$ & Mean $\pm S D$ & $\begin{array}{l}\mathrm{P} \\
\text { value }\end{array}$ \\
\hline T status & & 0.002 & & 0.067 & & 0.002 & & 0.001 \\
\hline $1 / 2$ & $11.72 \pm 2.78$ & & $7.28 \pm 1.54$ & & $120.50 \pm 126.64$ & & $13.90 \pm 14.71$ & \\
\hline 3 & $16.94 \pm 5.58$ & & $10.04 \pm 3.49$ & & $162.51 \pm 105.07$ & & $17.75 \pm 10.42$ & \\
\hline 4 & $23.23 \pm 3.95$ & & $14.88 \pm 3.33$ & & $381.33 \pm 102.50$ & & $42.45 \pm 8.66$ & \\
\hline $\mathrm{N}$ status & & 0.212 & & 0.140 & & 0.419 & & 0.647 \\
\hline No & $14.38 \pm 5.54$ & & $8.49 \pm 3.30$ & & $139.83 \pm 106.78$ & & $19.23 \pm 12.66$ & \\
\hline $\mathrm{N} 1$ & $16.39 \pm 5.46$ & & $10.27 \pm 4.37$ & & $160.68 \pm 98.61$ & & $19.23 \pm 12.95$ & \\
\hline N2 & $19.78 \pm 5.51$ & & $11.69 \pm 2.82$ & & $240.99 \pm 158.89$ & & $24.82 \pm 13.95$ & \\
\hline M status & & 0.244 & & 0.000 & & 0.573 & & 0.836 \\
\hline M0 & $15.03 \pm 5.37$ & & $8.51 \pm 2.72$ & & $163.30 \pm 122.55$ & & $17.54 \pm 11.93$ & \\
\hline M1 & $19.45 \pm 5.56$ & & $12.56 \pm 3.79$ & & $202.84 \pm 135.50$ & & $23.11 \pm 15.12$ & \\
\hline Differentiation & & 0.112 & & 0.120 & & 0.108 & & 0.612 \\
\hline Well & $10.35 \pm 0.07$ & & $7.10 \pm 1.13$ & & $55.30 \pm 40.30$ & & $4.35 \pm 0.07$ & \\
\hline Moderate & $16.13 \pm 5.27$ & & $9.77 \pm 3.72$ & & $162.19 \pm 107.04$ & & $19.39 \pm 12.53$ & \\
\hline Poorly & $21.29 \pm 6.22$ & & $12.39 \pm 3.29$ & & $283.49 \pm 165.35$ & & $25.59 \pm 15.81$ & \\
\hline
\end{tabular}

Figures 


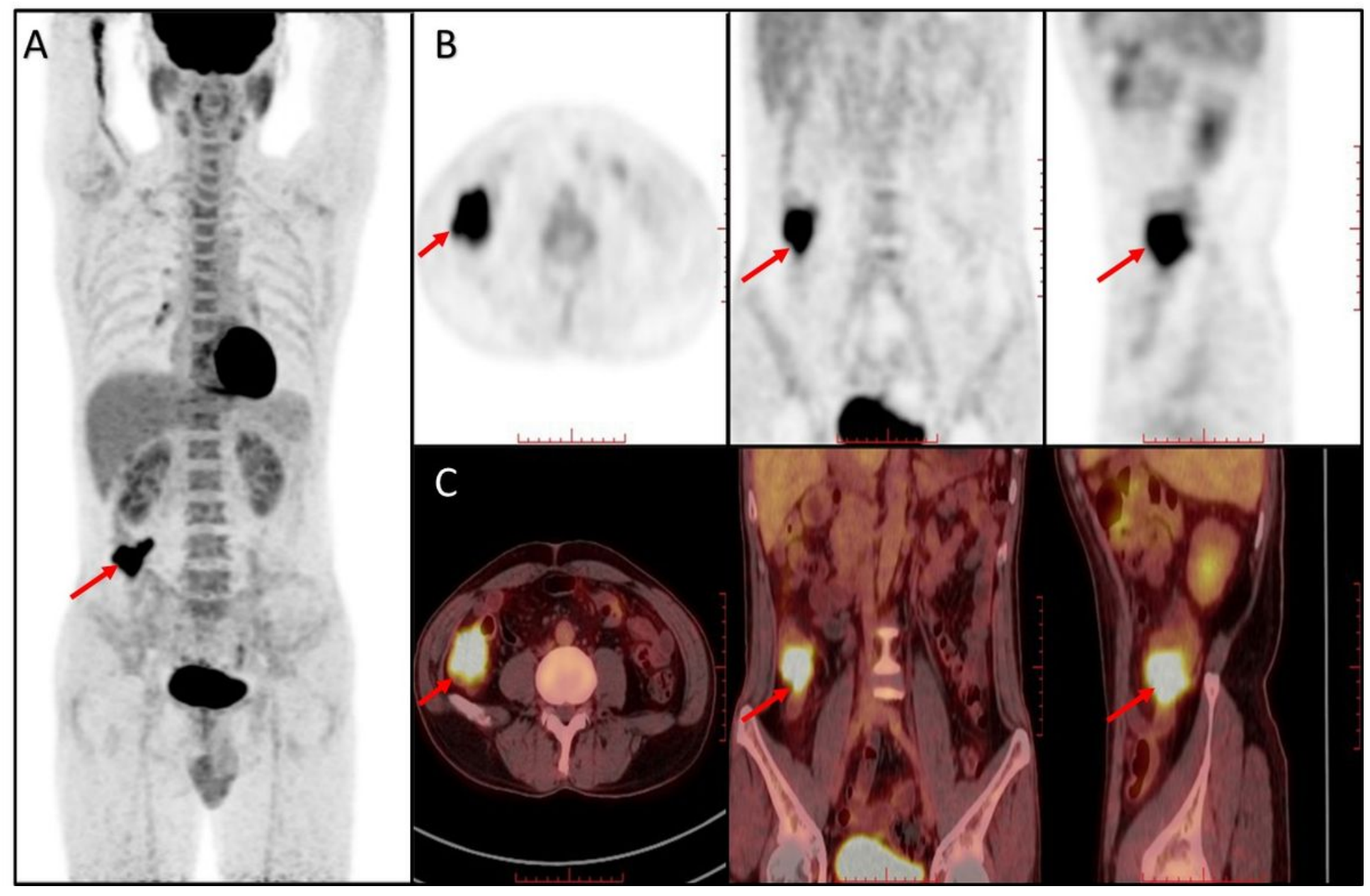

Figure 1

18F-FDG PET/CT image of CRC patient. A: Multiple intensity projection (MIP) of 61 year old male with CRC in T3 stage. $B$ : The uptake of FDG show in PET image with cross axial, coronary and sagittal position. C: The uptake of FDG show in PET/CT image with cross axial, coronary and sagittal position. (SUVmax: 9.7, SUVmean: 6.2, TLG: 132.3, MTV: 21.4). Red arrows indicate the uptake of FDG in tumor lesions. 


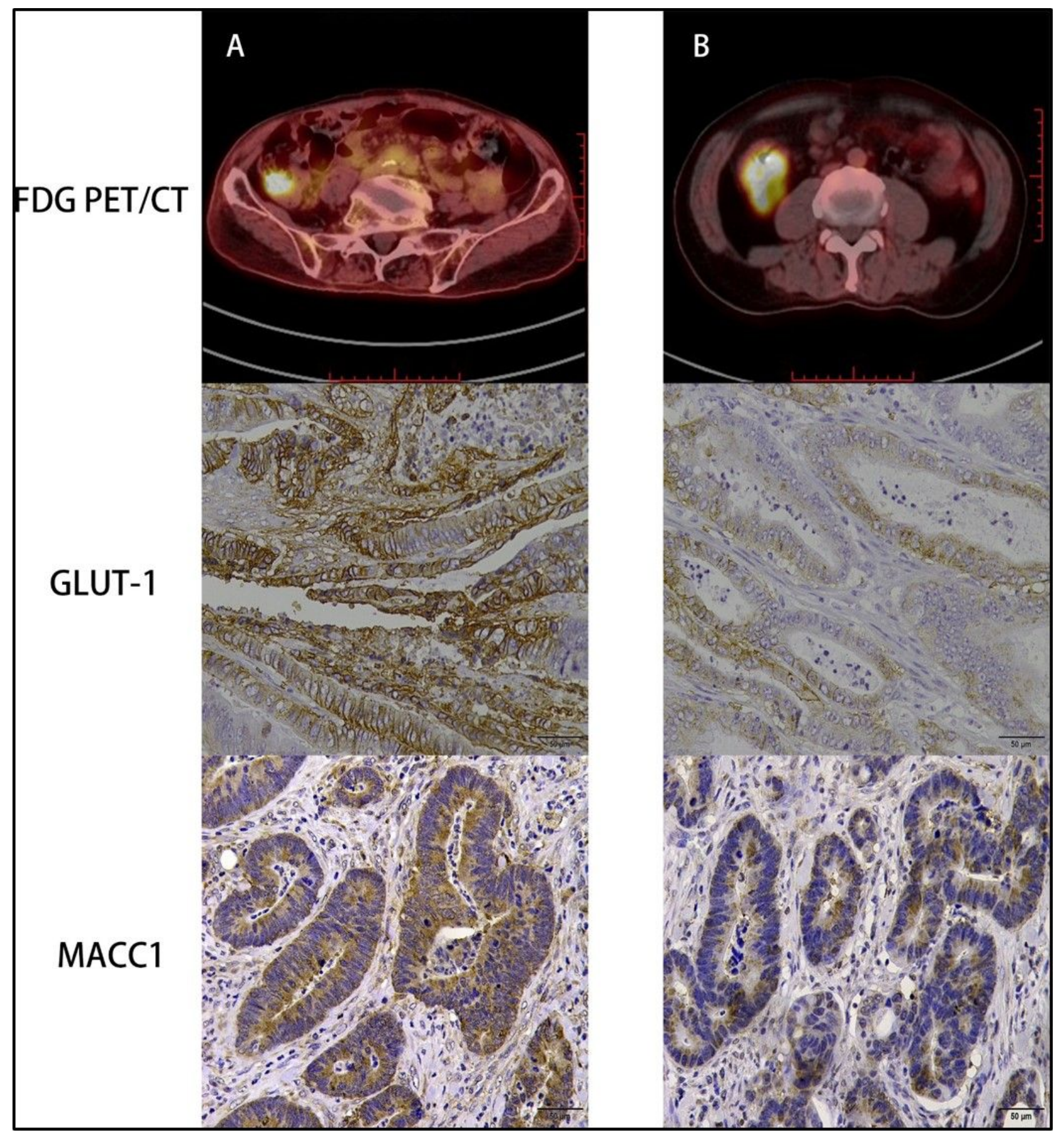

\section{Figure 2}

The expression level of GLUT-1 and MACC1 in CRC patients with different TNM stage. A: A 87 year old male with CRC in T3, N2 and M1 (liver metastases) stage, show a high expression of GLUT-1 and MACC1 in CRC specimen. B: A 55

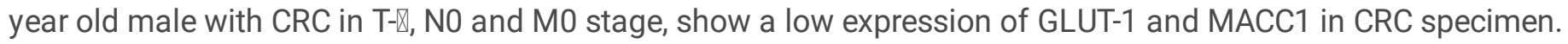

